Russie - Empire russe - Union soviétique et États indépendants

$53 / 4 \mid 2012$

Varia

\title{
Golfo Alexopoulos, Julie Hessler, and Kiril Tomoff, eds., Writing the Stalin Era
}

Jean-Paul Depretto

\section{OpenEdition}

\section{Journals}

Édition électronique

URL : http://journals.openedition.org/monderusse/7807

DOI : $10.4000 /$ monderusse. 7807

ISSN : $1777-5388$

Éditeur

Éditions de l'EHESS

\section{Édition imprimée}

Date de publication : 15 décembre 2012

ISSN : $1252-6576$

Référence électronique

Jean-Paul Depretto, « Golfo Alexopoulos, Julie Hessler, and Kiril Tomoff, eds., Writing the Stalin Era », Cahiers du monde russe [En ligne], 53/4 | 2012, mis en ligne le 09 septembre 2013, Consulté le 25 septembre 2020. URL : http://journals.openedition.org/monderusse/7807 ; DOI : https://doi.org/ 10.4000/monderusse.7807

Ce document a été généré automatiquement le 25 septembre 2020.

(c) École des hautes études en sciences sociales 


\section{Golfo Alexopoulos, Julie Hessler, and Kiril Tomoff, eds., Writing the Stalin Era}

Jean-Paul Depretto

\section{RÉFÉRENCE}

Golfo AleXopoulos, Julie Hessler, and Kiril Tomoff, eds., Writing the Stalin Era. Sheila Fitzpatrick and Soviet Historiography. New York : Palgrave Macmillan, 2011, 246 p.

1 Les travaux de l'historienne Sheila Fitzpatrick font partie du savoir commun de la profession : qu'on pense à des thèmes comme les vydvizhentsy ou la Stalinist soslovnost'. Certains ont même affirmé l'existence d'une "école Fitzpatrick», comme on a pu parler d'une " école Haimson »1. Quoi qu'il en soit, en 2006 un hommage lui a été rendu lors d'une conférence à l'université de Melbourne qui a réuni la plupart de ses ancien(ne)s doctorant(e)s. Beaucoup des essais qui composent ce livre sont issus de cette rencontre. Neuf des onze auteurs ont préparé leur thèse sous sa direction, à Austin (Texas) et Chicago.

2 Ce livre vise à dire la dette de tous les contributeurs à l'égard de la chercheuse, de la conseillère et de l'amie qu'a été ce professeur exceptionnel: il cherche à la fois à célébrer son œuvre et à éclairer sa personnalité. Comme les trois éditeurs le reconnaissent dans la préface, il implique évidemment une dimension affective.

3 L'ouvrage comporte douze chapitres, divisés en trois parties : il commence par deux textes consacrés aux travaux scientifiques de Sheila Fitzpatrick. Suivent neuf chapitres consacrés à des sujets divers, reliés de façon plus ou moins étroite à ses centres d'intérêt. Le dernier chapitre rassemble des souvenirs de personnes qui l'ont connue à des titres divers.

4 Le premier chapitre est dû à Ronald Suny, qui fut le collègue de Fitzpatrick à Chicago. Selon lui, l'œuvre de l'historienne est caractérisée par «l'engagement détaché »: elle 
respecte les standards les plus élevés d'objectivité, de neutralité et de fidélité aux sources; en même temps, elle défie sans crainte les orthodoxies. La froideur à l'égard de l'URSS et du marxisme est ici une constante: Fitzpatrick adopte une attitude critique vis-à-vis du régime soviétique, mais refuse la haine viscérale de guerre froide, ce qui lui sera reproché par les partisans de la théorie du totalitarisme. Elle évite l'idée d'une clef magique, d'un facteur essentiel qui expliqueraient les complexités changeantes de l'histoire soviétique. Son œuvre se construit à partir des sources, et non à partir d'une grande théorie ou d'une téléologie : elle est très souvent ethnographique. Ce qui la caractérise au premier chef, c'est l'engouement pour les archives, ce qui n'empêche pas le recours aux notions issues des sciences sociales.

Julie Hessler (chapitre 2) suit la trajectoire intellectuelle de Fitzpatrick en rapport avec quelques-uns de ses intérêts durables: le rôle de la classe sociale dans l'histoire soviétique ; les liens entre individu et société et entre individu et État dans la période de la révolution et sous Stalin; la culture soviétique ; l'intelligentsia et sa relation au régime bolchevik. La question spécifique qui a préoccupé Fitzpatrick dans les années 1970 était celle des soutiens des bolcheviks : à la fin des années 1920 et au début des années 1930, la promotion massive de vydvizhentsy a créé une base qui s'identifiait au régime. Cette enquête sur les vydvizhentsy a formé la base d'une défense beaucoup plus large de "l'histoire par en bas ». Depuis les années 1980, l'historienne est préoccupée par la relation entre l'individu et le collectif comme sujets de l'histoire. Dans ce tandem, elle voit de plus en plus l'individuel comme foyer de l'histoire sociale et finit, dans Tear Off the Masks! ${ }^{2}$, par adopter une approche microscopique de la société soviétique, avec des chapitres consacrés à un seul individu ou à un très petit groupe. Ces individus agissent en fonction de leurs intérêts.

6 Parmi les autres textes, je me contenterai d'en sélectionner quelques-uns, en fonction de mes compétences et de mes centres d'intérêt. Le remarquable essai de Matthew J. Payne sur la sédentarisation des nomades kazakhs (1928-1934) part d'une énigme : la combinaison de la promotion de la nationalité kazakhe et du massacre ethnique. La volonté d'« étatiser » les nomades n'explique guère le caractère " schizophrénique » de cette politique. Le rôle des cadres locaux est crucial pour expliquer la sédentarisation forcée : ils n'étaient pas des "courroies de transmission " passives des directives du Centre, mais des initiateurs actifs de campagnes pour atteindre les buts de Moscou. La sédentarisation doit être vue comme une dialectique complexe entre le régime et ses cadres qui, loin d'être des agents aveugles de l'État, veillaient à agrandir leur propre pouvoir. Au total, malgré les intentions officielles, cette sédentarisation est devenue une machine à transformer des nomades indépendants en vagabonds paupérisés.

7 Lynne Viola explore les récits autobiographiques des survivants des colonies spéciales; ces récits racontent l'histoire d'un "archipel du Goulag " peuplé de paysans. L. Viola examine les thèmes de ces écrits: la vie au village avant la collectivisation, la dékoulakisation, la vie dans les colonies spéciales, marquée par le rôle central du travail et la faim; l'autorité des commandants, 1937. L'auteur note que l'école est présentée sous un jour positif, car elle incarnait une possibilité de vie normale, d'avenir. Beaucoup de ces témoins étaient d'ailleurs des enseignants, des médecins et des vétérinaires à la retraite : on comprend pourquoi l'éducation joue un rôle si central dans leurs souvenirs.

8 Mark Edele part d'une initiative du gouvernement soviétique : en 1978, les participants à la guerre de 1941-1945 deviennent une catégorie privilégiée. Cette décision est 
consécutive à la pression qu'exercent les anciens combattants dans le cadre de la discussion du projet de nouvelle constitution (1977) : ils écrivent notamment des lettres au pouvoir. Nous sommes ici en présence d'une "action de masse non coordonnée " (p.124) que ce groupe est capable de réaliser, alors même que ses membres n'en sont pas conscients. M. Edele emploie le terme de "groupe d'ayants droit» (entitlement group) pour désigner «une accumulation d'individus qui partagent un sentiment similaire de mériter un traitement spécial et un respect spécial des autres membres de la société » (p. 123). Ce sentiment partagé conduisait les anciens combattants à agir de façon semblable dans certaines situations : ils se comportaient comme s'ils étaient un groupe, une nouvelle entité sociale née de la guerre, mais que le pouvoir central n'avait jamais voulu créer. Ils furent finalement cooptés dans le système de groupes de statut construit par l'État soviétique et en échange firent preuve de loyauté.

Kiril Tomoff analyse la bataille menée, aux États-Unis et en France, par les dirigeants soviétiques pour obtenir le retrait des écrans de The Iron Curtain (1948) du réalisateur William A. Wellman, présenté comme le premier film de guerre froide tourné à Hollywood. Selon l'auteur, la politique de l'URSS est particulièrement importante pour notre compréhension du monde actuel, car la confrontation culturelle entre la Russie soviétique et les États-Unis a modelé le développement des relations internationales après 1989. La stratégie soviétique dans cette bataille révèle une confiance surprenante envers des amis de l'étranger pour défendre les intérêts de l'URSS, un haut degré de souplesse pratique dans la poursuite de buts idéologiques et une certaine disposition à combattre l'Occident dans les termes de ce dernier. De petits fonctionnaires soviétiques ont joué un rôle actif dans ces batailles où l'URSS a souvent réussi à court terme. Toutefois, l'acceptation des termes occidentaux du conflit a finalement été fatale.

En rassemblant des souvenirs dans le dernier chapitre, les éditeurs ont voulu apporter une contribution à un portrait de Fitzpatrick. Le résultat, à mon avis, est inégal et les textes ne sont pas tous convaincants. Le lecteur lira avec intérêt le témoignage de l'ancien mari de Sheila, Jerry F. Hough, qui expose ses vues sur leur influence intellectuelle réciproque dans les années 1970 et 1980 et sur le climat intellectuel de la soviétologie à cette époque. L'historien Vladimir Kozlov raconte sa rencontre avec Fitzpatrick, en la replaçant de façon passionnante dans son itinéraire personnel et dans le contexte de l'historiographie russe. Katerina Clark, spécialiste de littérature, évoque avec sensibilité son amie Sheila, qui, comme elle, est une Australienne expatriée dans l'Université américaine. Il est émouvant d'apprendre qu'après un deuil, Sheila a trouvé une consolation dans la pratique du violon ou que dans l'Australie des années de guerre froide, elle a été ostracisée à l'école à cause du "communisme " supposé de son père Brian Fitzpatrick. Mais ces souvenirs sont parfois trop indiscrets à mon goût sur des faits relevant de la vie privée. D'autre part, les extraits (p. 203-209) de la correspondance entre Sheila et son frère David (1968-1979) ne me semblent pas apporter beaucoup: je ne vois d'ailleurs pas en quoi elle y apparaît comme « arrogante» (p. 202), comme l'affirment les éditeurs du livre.

11 Bien que j'aie terminé sur une note négative, il faut souligner que cet hommage amplement mérité à une grande historienne est une lecture passionnante pour tout spécialiste de l'URSS : nous avons tant appris d'elle! 


\section{NOTES}

1. Cf. le débat à ce sujet dans Acta Slavica Iaponica, 24 (2007), p. 229-241: « Roundtable: What is a School? Is There a Fitzpatrick School of Soviet History? ", avec la participation de David Wolff, Jonathan Bone, Mark Edele, Matthew Lenoe et Ronald Grigor Suny.

2. Tear Off the Masks!: Identity and Imposture in Twentieth-Century Russia, Princeton University Press, 2005. 